\title{
A Cellular-Assisted Efficient Handover Algorithm for Wireless Sensor Networks
}

\author{
Jun Zhu', Hui Gao ${ }^{1,2}$, Yuling Ouyang ${ }^{2}$ \\ ${ }^{1}$ Key Laboratory of Intelligent Computing \& Singnal Processing, Ministry of Education Anhui University, Hefei, China \\ ${ }^{2}$ Shanghai Research Center for Wireless Communications, Shanghai, China \\ Email: junzhu@ahu.edu.cn, gaohuixy4869@163.com,yuling.ouyang@shrcwc.org
}

Received August 10, 2012; revised September 15, 2012; accepted September 25, 2012

\begin{abstract}
Heterogeneous network convergence and handover have become very hot in recent years. This paper proposed an efficient handover scheme in Multi-PAN Wireless Sensor Networks (WSNs). A number of edge nodes are set at the edge of each Personal Area Networks (PANs). A user equipment (UE), which has WSN and cellular network interface, acts as sensor node or mobile cluster head in WSN area. Thus, edge early warning can be acquired from edge nodes and neighbor channel information can be acquired with BS-assistance. Simulation results show that low transmission interrupted delay and low energy consumption can be achieved compared with conventional scheme in WSN.
\end{abstract}

Keywords: Efficient Handover; Edge Early Warning; Special Beacon; Wireless Sensor Networks

\section{Introduction}

With the development of WSNs [1-3] and cellular networks, modern technologies have been prepared for creating the convergent communication infrastructures, through which WSNs and cellular networks convergence have become inevitable. These heterogeneous networks appear in many application areas [4], especially in the health care. In these applications it is usually needed for mobile sensor nodes to frequent handover and to transmit data between Multi-PANs.

If sensor nodes move between Multi-PANs, the nodes are required to scan all channels to find the channel of nearby PAN which sensors move to, and then hand over to the target transmission channel. Because of scanning all channels, it will not only bring energy cost, but also will interrupt the data transmission for a long time and influence the transmission delay and QoS. So how to use the UE or cellular network assisted to realize and optimize efficient handover between Multi-PANs in Zig-Bee wireless sensor network is a key problem to be solved. And because sensor nodes have limited energy and are not convenient to replace battery, how to reduce energy consumption is also an important problem for WSN (e.g., SMAC [5], TMAC [6]).

There are some handover protocols in WSN. Standard ZigBee handover is in specification IEEE 802.15.4 [7] and ZigBee specification [8,9]. If an associated node wants to leave the PAN, it shall send a disassociation notification command to its coordinator. Sensor node scans all channels from lowest channel number to the highest in order with peak energy. For the duration of the scan, the node shall suspend beacon transmission and data transmission. Until selecting a channel of nearby PAN which it wants to move, the mobile sensor sends an association request command to the coordinator of the PAN. Richard et al. in [10] proposed a handover mechanism for WSNs. Sensor nodes can hand over in certain areas to achieve connecting from a mobile cluster head to a fixed and main-powered cluster head which connected to a permanent power supply for saving energy. The mobile sensor, mobile cluster head and fixed cluster head are on the same channel. It however doesn't consider sensor nodes moving in different PANs and channels scenario.

This paper proposed an efficient handover scheme in Multi-PAN WSN. In the scheme, each PAN has one channel for communication in WSN area. UE and mobile nodes form a mobile cluster, they move in one PAN and attach to the fixed node to communicate. The mobile nodes have to transmit data continuously wherever they move. When the mobile nodes move from one PAN to another, they will trigger handover. Through coordination of the BS, edge nodes can be set at the edge of PAN to achieve edge early warning and UE can acquire the neighbor PAN channel information with cellular link to achieve efficient handover and reduce transmission interrupted delay.

The remainder of this paper is organized as follows. Section 2 introduces the scenario and the efficient hand- 
over scheme we proposed in detail which includes edge node decision algorithm and procedure of the proposed scheme. Section 3 is the results and analysis of the simulation. Section 4 concludes this paper.

\section{Efficient Handover Algorithm}

\subsection{System Structure}

The efficient handover scheme is based on the scenario which is shown in Figure 1. We consider the convergent scenario for wireless sensor network (WSN) and cellular network. In the cellular system, the user equipments (UEs) are under the control of a cellular base station (BS). In the coverage area of a cellular network, there also exists a group of wireless sensor nodes composing the WSN networks. In the convergent scenario, the cellular UEs are dual-mode and have both WSN and cellular interfaces, and they can act as mobile cluster heads for the WSN. The UEs can provide backhaul access for the WSN nodes under the help of 3GPP-LTE BS. The WSN is based on Multi-PANs topology for beacon-enabled mode. Each PAN has one channel for communication. Some sensor nodes and UEs are moving in the WSN area but connect with the WSN all the time. WSN control unit (e.g., BS) keeps the topology of WSN and knows coverage area of all PANs and overlapping area of PANs.

\subsection{Edge Node Decision Algorithm}

Edge node is located at the edge of its PAN or in the overlapping area of two PANs, and it is used to the edge early warning for mobile sensors. The edge node is selected by BS after organized the WSN or after topology and usually has strong communication ability.

Edge node sets two beacon formats. If there is no mobile node, edge node will send the normal beacon. If there are some mobile nodes, edge node will be triggered and send the special beacon containing edge flag. In beacon payload, the bit 16 and 17 are reserved. So we add edge flag in beacon payload which occupied 1 bit, which is shown in Table $\mathbf{1}$ [7] and Table 2.

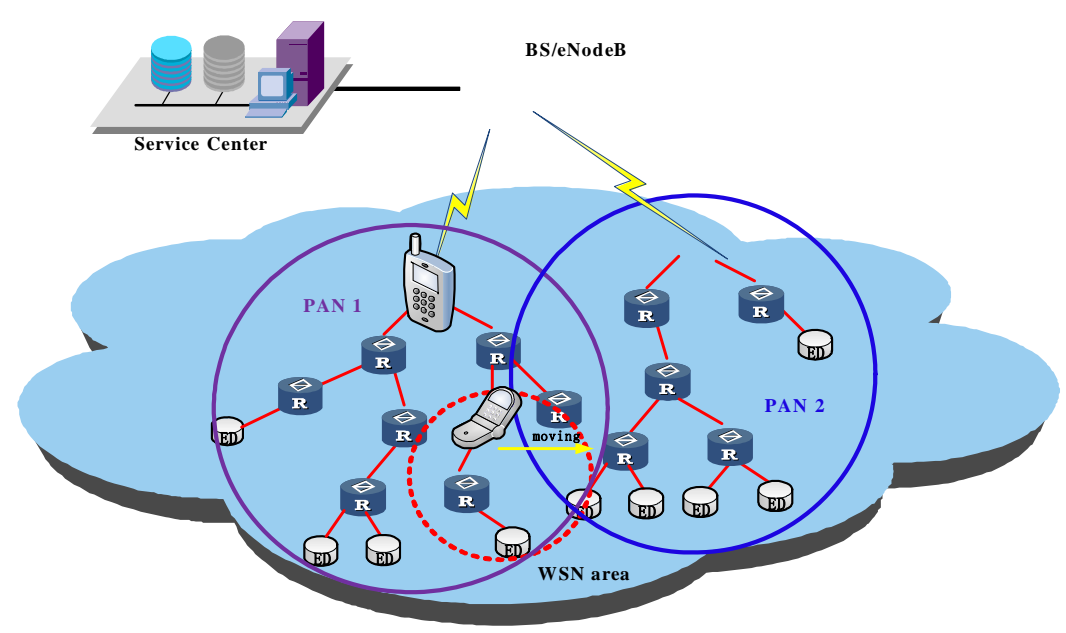

Figure 1. System application scenario.

Table 1. Beacon frame format of edge node.

\begin{tabular}{ccccc}
\hline \multirow{2}{*}{ MHR } & \multicolumn{4}{c}{ MAC Payload } \\
\cline { 2 - 5 } & Octets: 2 & Variable & Variable & Variable \\
& Superframe Specification & GTS fields & Pending Address fields & Beacon Payload \\
\hline
\end{tabular}

Table 2. Special beacon payload format of edge node.

\begin{tabular}{ccccc}
\hline Bits: $0-7$ & $8-11$ & $12-15$ & 16 & 17 \\
\hline Protocol ID & Stack profile & Nwk Protocol version & Edge flag & Reserved \\
Bits: $19-22$ & 23 & $24-87$ & $88-111$ & Router capacity \\
Device depth & End device capacity & Nwk extended & Tx offset \\
PANID & (optional) & nwkUpdateID \\
\hline
\end{tabular}


During the network initial phase, each Gateway (GW) obtains approximately location of each sensor node, forms the topology of PAN and reports the information to BS. BS saves the approximately location, range and neighbor PAN information of all PANs and uses the related information of PANs to determine the edge node. The steps are as follows.

BS searches the nodes of neighbor PAN at the double transmission range of node in one PAN (e.g. PAN 1) and saves the nodes of neighbor PAN in edge neighbor node list. Then BS determines node in edge neighbor node list as candidate edge node of PAN 1 and selects the edge node from candidate edge node in PAN 1. If the distance between two candidate edge nodes in the same PAN is below the threshold, BS will select one node as edge node; If BS will select both nodes as edge nodes. After determining all edge nodes in Multi-PAN area, BS will send edge-set signaling to inform them.

\subsection{Edge Node Information Table}

When all edge nodes are selected, BS saves a PAN channel information table including PAN ID, edge node ID, location, channel and neighbor channel. According to the information in the PAN channel table, BS knows which neighbor channel the edge node has. The edge node information table is shown in Table 3. The detailed parameters in this table include but not limited to below elements:

- PAN ID is the 16-bit address of a PAN in WSN network.

- Edge node ID is the 16-bit network address of a BSselected edge node in WSN.

- Edge node location is the approximate location information of the edge node in a PAN.

- Edge node neighbor channel is the neighbor PAN communication channel of the edge node.

\subsection{Procedure of Efficient Handover Algorithm}

As mentioned above, we used an efficient handover method in Multi-PAN WSN. The complete procedure is as follows:

Step 1: When organizing the WSN, BS determines which sensor to be edge node and informs them with edge-set signaling via WSN links.

Step 2: When UE and its mobile cluster move to the edge of PAN, its topology will be changed and its parent node will change into edge node. At the same time, edge node discovers that there is mobile node connecting to it. So the edge node is triggered and sends the special beacon periodically.

Step 3: UE receives the special beacon containing edge flag, which is edge early warning for mobile cluster. When the special beacon is received by mobile node of mobile cluster, it will report the information to UE.

Step 4: UE sends request containing PAN ID and edge node ID about channel information of neighbor PAN to BS with cellular interface. BS queries the neighbor channel table and replies neighbor PAN channel information and to UE with cellular interface.

Step 5: UE informs each node of mobile cluster about neighbor PAN neighbor channel information. The mobile cluster scans the obtained neighbor channel and handovers.

All the procedure of efficient handover scheme is described in Figure 2.

Table 3. Edge node information table.

\begin{tabular}{llll}
\hline PAN ID & Edge node ID & $\begin{array}{l}\text { Edge node } \\
\text { location }\end{array}$ & $\begin{array}{l}\text { Edge node } \\
\text { neighbor channel }\end{array}$ \\
\hline
\end{tabular}

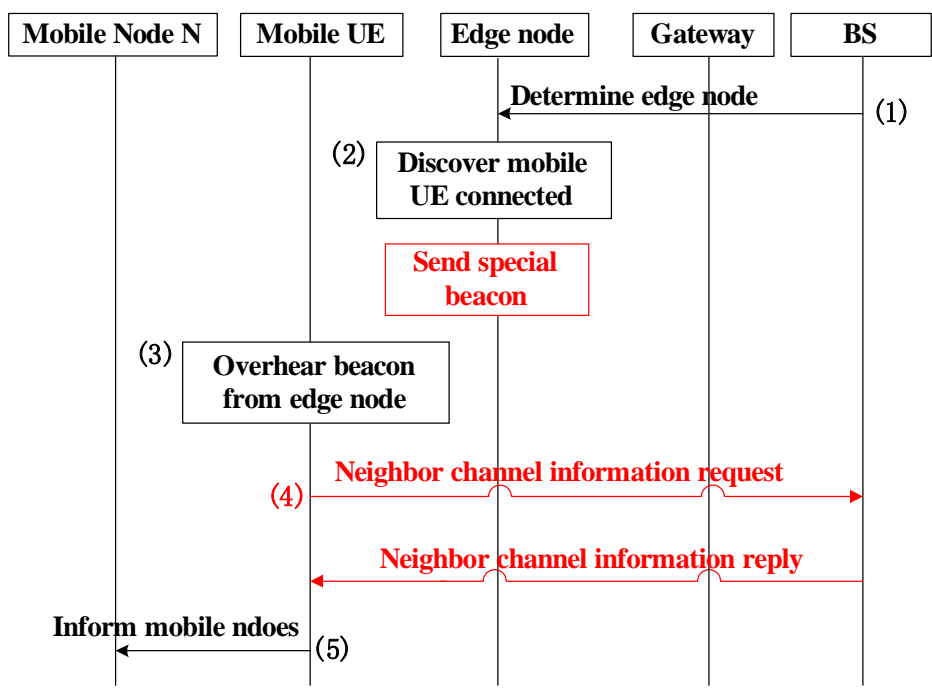

Figure 2. Signaling for procedure of efficient handover scheme. 


\section{Analysis and Simulation}

\subsection{Analysis of Efficient Handover Algorithm}

WSNs always have the question of energy consumption. Devices will be battery powered, and battery replacement or recharging in relatively short intervals is impractical. Therefore, the power consumption is of significant concern. Energy of cellular networks has large advantage comparing with WSN. So it's a great improvement for WSN to transfer energy consumption from the sensor networks to the cellular networks.

The energy consumption of conventional method in WSN is included in Equation (1):

$$
P_{\text {handover_wsn }}=M \times P_{\text {request }}+N \times P_{\text {scan }}+M^{\prime} \times P_{\text {request }}^{\prime}
$$

- $P_{\text {request }}$ means the energy consumption that sensor node which has disassociation with its coordinator, would send request signaling to its original channel for association again. $M$ is the hop number of request signaling which is sent to original coordinator.

- $P_{\text {scan }}$ means the energy consumption that sensor node which has disassociation with its coordinator, scans each channel from lowest number to highest in order to find a appropriate one to association. The device is expected to scan in 16 channels. $N$ is the number of scanning channels.

- $P_{\text {request }}^{\prime}$ means the energy consumption that sensor node which has found a appropriate channel, would send a association request to the coordinator. $M^{\prime}$ is the hop number of request signaling which is sent to new coordinator.

Thus the energy consumption of proposed method is included in Equation (2):

$$
P_{\text {handover_new }}=P_{\text {edge_inform }}+P_{U_{E_{-}} \text {request }}+I \times P_{\text {scan }}
$$

- $P_{\text {edge_inform }}$ means the energy consumption that eNB sends edge-set signaling to inform the edge node.

- $P_{\text {UE_request }}$ means the energy consumption that UE sends neighbor channel request with edge node ID to eNB for itself or mobile cluster.

- $P_{\text {scan }}$ means the energy consumption that sensor node scans the target channel for only one superframe period. $I$ is the number of mobile nodes in mobile cluster.

According to the calculation, $N^{*} P_{\text {wsn }}>P_{\text {new }}(N>4)$. The proposed method is suitable for frequent handover or mobile cluster handover. The proposed method would be used and guarantee the power consuming in the cellular link should be smaller than normal sensor network routine.

When sensor node moving in the WSN area, transmission with less interrupted delay is also very important.

The time spent on handover in conventional method is shown in Equation (3):

$$
T_{\text {handover_wsn }}=M \times T_{\text {request }}+N \times T_{\text {scan }}+M^{\prime} \times T_{\text {request }}^{\prime}
$$

- $T_{\text {request }}$ means the time spent on sending a association request to the original coordinator. $M$ is the hop number which signaling sent to the original coordinator.

- $T_{\text {scan }}$ means the time spent on scanning each channel, which is [a Base Super frame Duration $\times(2 n+1)$ ] symbols, where the $n$ is the value of the Scan Duration parameter, which at the ranges from 0 to 14 . It means scanning each channel will spent $8 \mathrm{~ms}$ to 200 $\mathrm{ms}$, which is a long time for transmission interruption. And the number of scanning channel $N$ is random.

- $T_{\text {request }}^{\prime}$ means the time spent on sending a association request to the new coordinator. $M$ ' is the hop number which signaling sent to the new coordinator.

The time spent on handover in proposed method is shown in Equation (4):

$$
T_{\text {handover_new }}=T_{U E_{-} \text {request }}+T_{\text {eNB_reply }}+T_{\text {scan }}+T_{\text {request }}^{\prime}
$$

- $T_{U E_{-} \text {request }}$ means the time UE sends a neighbor channel request with edge node ID to eNB for itself or mobile cluster.

- $T_{\text {eNB_reply }}$ means the time eNB sends neighbor channel response signaling back to UE.

- $T_{\text {scan }}$ means the time spent on scanning one channel.

- $T_{\text {request }}^{\prime}$ means the time spent on sending a association request to the coordinator.

A signaling interactive time is about $3 \mathrm{~ms}-5 \mathrm{~ms}$ [11] on the physical layer of air interface in TD-LTE. According to the above process, connection time is about 9 ms - 15 ms which calculating the number of signaling interaction. The transmission date time is $4 \mathrm{~ms}-6 \mathrm{~ms}$ (two child frame length), so the consuming time is $13 \mathrm{~ms}$ - $21 \mathrm{~ms}$ in cellular, which $18 \mathrm{~ms}$ being typical.

According to the calculation, $T_{\text {handover_wsn }}>T_{\text {handover_new, }}$, the proposed method has reduced a large of interrupted transmission delay. UE would be used and guarantee the time consuming in the cellular link should be smaller than normal sensor network routine.

\subsection{Simulation Scenario Description}

To evaluate the effectiveness of efficient handover scheme, we measure the power consumption and average scanning delay when mobile nodes hand over. We compare the results of our proposed scheme and traditional scheme. Here we take standard ZigBee handover as traditional scheme. For the convenience of analysis, some assumptions are as follow:

- There are 2 numbers of PANs. In each PAN, there are 5 mobile nodes and a UE forming a mobile cluster and $\mathrm{N}$ numbers of static sensor nodes.

- The number of channel is 16 (ZigBee).

- The scanning delay of each PAN channel is $30 \mathrm{~ms}$. 
The number of scanning channels in conventional scheme is random.

\subsection{Analysis of Simulation Results}

We first measure the average scanning delay of our proposed scheme and traditional scheme.

The simulation results of latency are shown in Figure 3, where the plus marker represents for latency using traditional scheme, while circle marker represents the latency after using efficient handover scheme with BS assisted. It can be seen from the simulation results that the proposed scheme improves network performance for each handover.

We also measure the power consumption of our proposed scheme and traditional scheme.

The simulation results of power consumption are shown in Figure 4, where the plus marker represents for

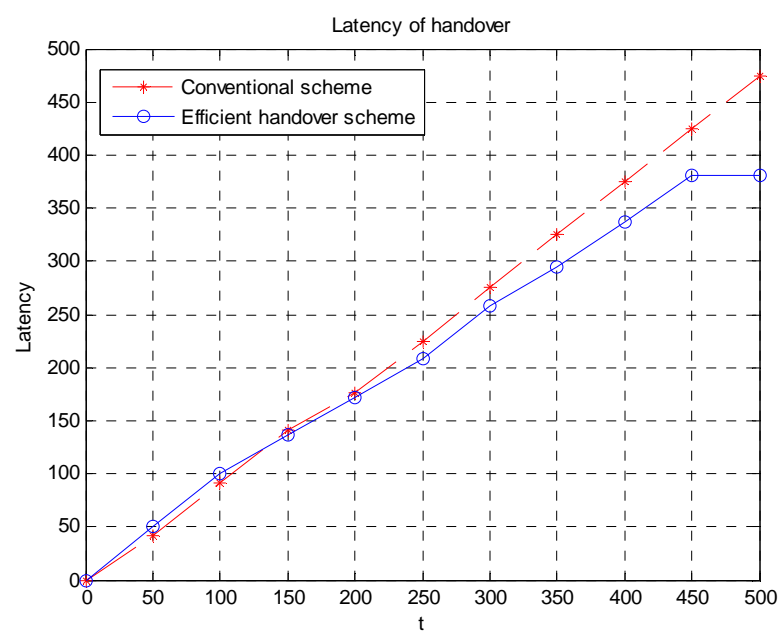

Figure 3. Performance comparisons with respect to average delay time.

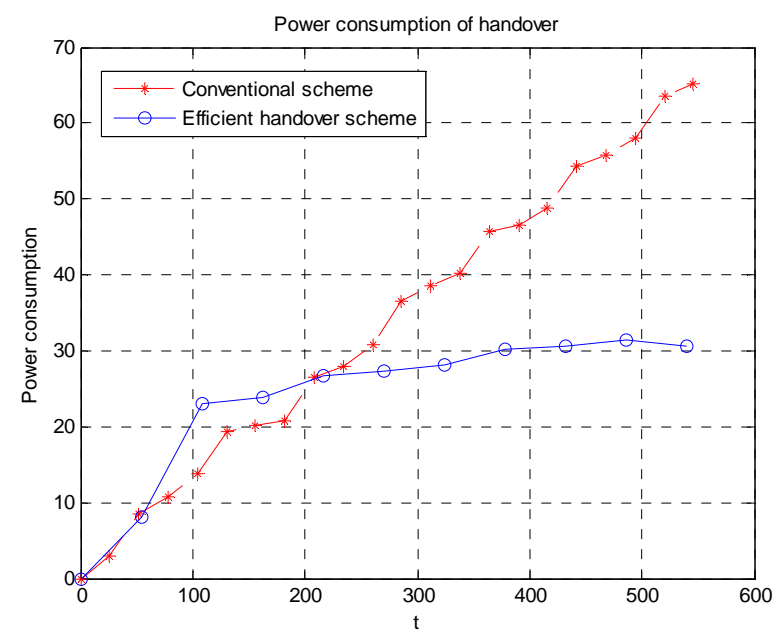

Figure 4. Performance comparisons with respect to power consumption. power consumption using traditional scheme, while circle marker represents power consumption using efficient handover scheme. It can be seen from the simulation results, the circle marker has a slow grow after the inflection point $t=100$, that because mobile nodes in the mobile cluster has received neighbor PAN channel information from UE and need not to scan all the channels for handover. So the addition of handover mechanism with BS assisted can help to reduce the handover power consumption of mobile cluster or frequent handover power consumption of mobile nodes. Because mobile nodes in mobile cluster will obtain the target PAN channel information instead of scanning channels to find an appropriate PAN.

\section{Conclusion}

In this paper, we propose an efficient handover mechanism in Multi-PAN area. We design the edge node used for edge early warning; then we use BS to manage the Multi-PAN channel information and use UE to acquire neighbor PAN channel information to reduce the scanning channel time and power consumption. The simulation results indicate that the presented scheme can reduce transmission interrupted delay and power consumption when mobile sensor nodes hand over in Multi-PAN under the help of cellular network.

\section{Acknowledgements}

This work was supported by the National Natural Science Foundation of China (No. 61071168) and the 211 Project of Anhui University, the Anhui Provincial Natural Science Foundation (No. 11040606Q06).

\section{REFERENCES}

[1] D. Culler, D. Estrin and M. Srivastava, "Overview of Sensor Networks,” IEEE Computer, Vol. 37, No. 8, 2004, pp. 41-49. doi:10.1109/MC.2004.93

[2] J. Yick, B. Mukherjee and D. Ghosal, "Wireless Sensor Network Survey,” Computer Networks, Vol. 52, No. 12, 2008, pp. 2292-2330. doi:10.1016/j.comnet.2008.04.002

[3] V. Rajaravivarma, Y. Yang and T. Yang, "An Overview of Wireless Sensor Network and Applications,” Proceedings of 35th Southeastern Symposium on System Theory, Morgantown, 22 April 2003, pp. 432-436.

[4] A. Wheeler, "Commercial Applications of Wireless Sensor Networks Using Zigbee,” IEEE Communications Magazine, Vol. 45, No. 4, 2007, pp. 70-77. doi:10.1109/MCOM.2007.343615

[5] W. Ye, J. Heidemann and D. Estrin, "Medium Access Control with Coordinated Adaptive Sleeping for Wireless Sensor Networks," IEEE/ACM Transactions on Networking, Vol. 12, No. 3, 2004, pp. 493-506.

[6] T. Van Dam and K. Langendoen, “An Adaptive Energy- 
Efficient MAC Protocol for Wireless Sensor Networks,” Proceedings of the 1st International Conference on Embedded Networked Sensor Systems, 2003, pp. 171-180. doi:10.1145/958491.958512

[7] IEEE-TG15.4, "Part 15.4: Wireless Medium Access Control (MAC) and Physical Layer (PHY) Specifications for Low-Rate Wireless Personal Area Networks (LR-WPANs)," IEEE Standard for Information Technology, New York, 8 September 2006.

[8] ZigBee Alliance, “ZigBee Specification,” 2007 ZigBee
Standards Organization, San Ramon, 2008.

[9] E. Karapistoli, F.-N. Pavlidou, I. Gragopoulos and I. Tsetsinas, "An Overview of the IEEE 802.15.4a Standard,” IEEE Communications Magazine, Vol. 48, No. 1, 2010, pp. 47-53. doi:10.1109/MCOM.2010.5394030

[10] R. M. Miller-Smith, "Handover Mechanism for Sensor Networks,” US2010/0285807 A1, 2010.

[11] R1-060932. CATT, RITT, TD-Tech, "EUTRA TDD Random Access Procedure,” Athens, 2006, pp. 27-31. 